\title{
Chain Length and Thermal Sensitivity of the Infrared Spectra of a Homologous Series of Anhydrous Silver(I) $n$-Alkanoates
}

\author{
Peter N. Nelson ${ }^{1,2}$ \\ ${ }^{1}$ Department of Chemistry, The University of the West Indies, Mona Campus, St. Andrew, Kingston 7, Jamaica \\ ${ }^{2}$ Department of Materials and Interfaces, Weizmann Institute of Science, P.O. Box 26, Rehovot, Israel \\ Correspondence should be addressed to Peter N. Nelson; chessnelo@gmail.com
}

Received 16 November 2015; Revised 2 February 2016; Accepted 4 February 2016

Academic Editor: Jin Zhang

Copyright (C) 2016 Peter N. Nelson. This is an open access article distributed under the Creative Commons Attribution License, which permits unrestricted use, distribution, and reproduction in any medium, provided the original work is properly cited.

\begin{abstract}
The thermal and chain length sensitivity of the infrared spectra of some solid state anhydrous silver(I) salts ( $n$-octanoate to $n$ eicosanoate, inclusive) are discussed. At ambient temperature, the tilted alkyl chains, anchored to the metal planes via chelating bidentate coordination to the silver ions, are crystallized in the fully extended all-trans conformation. Interestingly, though all compounds are crystallized in a monoclinic crystal system, their lateral chain packing, van der Waals effects, and hence vibrational features are chain length-dependent. This is a direct result of electrostatic effects of the COO group in addition to vibrational coupling between $\mathrm{CH}_{2}, \mathrm{CH}_{3}$, and $\mathrm{COO}$ modes, an effect which varies significantly with chain length. Variable temperature infrared measurements indicate significant irreversible changes in the metal-carboxyl coordination sphere, most likely due to bond fission. For long chain adducts $\left(n_{c}>12\right)$, thermally induced crystal system switching, monoclinic to triclinic, indicates greater thermal sensitivity of their alkyl chains. During heating, the regions of the hydrocarbon chains, furthest from the COO, become increasingly molten and mobile as the stepwise melt advances towards the solid COO moieties. This solid-liquid melting behaviour is responsible for mesophase formation in metal carboxylate systems.
\end{abstract}

\section{Introduction}

The ability of metal carboxylates to form three-dimensional self-assembled layered structures in addition to diverse coordination modes, in the solid state, is some of their most fascinating structural attributes. However, for most long chain adducts, single crystal data is sparse due to their low molecular symmetries which caused the significant flexibility of the alkyl chains; hence, they usually solidify as thin needle-like or twinned plate-lake crystals, unsuitable for single crystal diffraction. Nonetheless, crystal structures for various short chain homologues have been published [1-3]. For example, crystal structures for both silver(I) acetate [4] and propanoate [5] show carboxylate groups coordinated in a syn-syn type bridging bidentate mode to silver ions, forming dimeric units, held together via extensive interdimer electrostatic forces. Silver-silver distances in these units allowed for extensive metal-metal interactions and possibly bonding. However, though both crystal structures agreed on the type of metalcarboxyl bonding, their molecular and lattice packing were different. For instance, silver acetate was triclinic with $P 1$ symmetry whilst the monoclinic propanoate was of $P 2_{1} / a$ Bravais lattice. Indeed, this difference shows clearly that their molecular packing is chain length dependent but their coordination to the central metal ion is physically isostructural. Though further structural changes for longer chain adducts might be gradual, the sensitivity of vibrational spectroscopy to lattice packing provides an invaluable tool for assessing such changes. Surely, the industrial importance of silver $n$-alkanoates stemming from their wide spread use as low angle calibrants for neutron diffraction instruments [6] and as silver sources in photothermogravimetry (PTG) and photography [7-10], makes relevant a detailed study of their molecular and lattice packing, based on a method, nondestructive to sensitive intermolecular interactions. Furthermore, given the difficulties associated with obtaining single crystals for the long chain adducts, in addition to the destructive nature of X-rays, vibrational features associated with different molecular packing and intralattice interactions are significant. Also, since, like many amphiphilic systems, silver carboxylates exhibit 


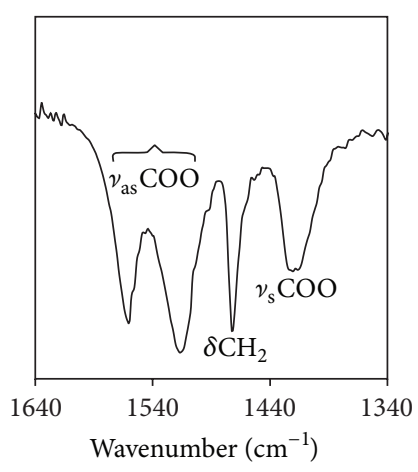

(a)

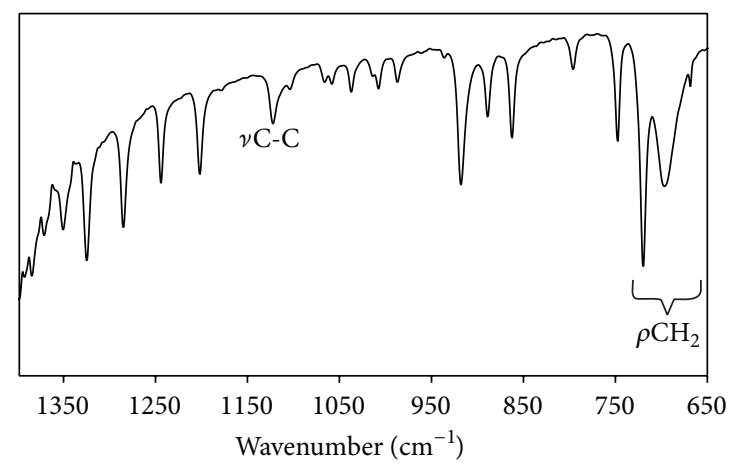

(b)

FIGURE 1: (a) Carboxyl stretching ( $\nu_{\mathrm{as}} \mathrm{COO}$ and $\left.\nu_{\mathrm{s}} \mathrm{COO}\right)$ and methylene bending mode $\left(\delta \mathrm{CH}_{2}\right)$ for $\mathrm{AgC} \mathrm{C}_{11}$. (b) The methylene twisting, rocking, and progression regions for $\mathrm{AgC}_{11}$.

various premelting phases when heated from the room temperature solid to the melt, some of which have been described as liquid crystalline [11], the effect on temperature on their lattice packing is of interest. For example, Nelson [12-14] and Binnemans et al. [11] showed that for silver carboxylates $\left(\mathrm{AgC}_{4} \cdots \mathrm{C}_{28}\right)$ the number of premelting phases increases with increasing chain length, the melt being at highest temperature, as is common for these compounds. However, the suggestion by Binnemans that more than one liquid crystalline phases were present in the phase sequences for some silver compounds was questioned by Nelson, based on high temperature microscopy data, but not totally ruled out. The difficulty associated with characterizing the phases of these compounds stems from the fact that they decompose at the melt-to-yield nanoclusters [15]. This suggests that they could possibly be applied in the solid state thermal synthesis of nanoparticles for various applications. Therefore, it is clear that the evolution of their structural features with chain length and temperature is of importance. Furthermore, since it has been recently proven by Berson and coworkers [16] that it is possible to electrochemically influence the growth of a silver nanowires on the surface of carboxyl terminated alkyl silane self-assembly monolayers in the absence of moisture, it is also clear that silver-carboxyl interactions are of significant technological importance.

Therefore, in this paper, the vibrational features of some anhydrous silver carboxylates $\left(\mathrm{AgC}_{8} \cdots \mathrm{C}_{20}\right)$ are discussed in detail and are correlated with their respective molecular and lattice packing interactions. Their structural evolution is also assessed as a function of chain length and temperature.

\section{Experimental}

The white microcrystalline solids were prepared by metathesis, that is, reaction of the sodium salt of the $n$-alkanoic acids (Aldrich, 95+\%), in ethanol, with an excess of aqueous silver nitrate (Aldrich, 99.9\%) at ca. $343 \mathrm{~K}$. The sodium salts were prepared by dissolving the relevant $n$-alkanoic acid in $c a$. $50 \mathrm{~cm}^{3}$ of ethanol which was then combined with $\mathrm{ca} .50 \mathrm{~cm}^{3}$ of ethanol containing a stoichiometric amount of sodium hydroxide (Aldrich, 99.9\%) whilst stirring. Subsequent to stirring the aforementioned mixture for a $30 \mathrm{~min}$., an aqueous solution of silver nitrate was added, drop-wise with stirring, to the as prepared sodium $n$-alkanoate solution. This resulted in immediate precipitation of a white solid. After stirring for a further $30 \mathrm{~min}$., at ambient temperature, the precipitate was collected by filtration, washed thrice with a solution of $1: 1$ acetone-methylene chloride mixture, and vacuum dried for $24 \mathrm{~h}$. The compounds were then stored in the dark (because of their sensitivity to light), in a desiccator over silica gel. Calculated yields were in excess of $90 \%$. C-H analysis, reported elsewhere [12], proves that the compounds are of high purity.

Infrared spectra were recorded from ground samples, deposited on a germanium window of a Bruker Tensor 37 ATFT IR spectrometer in the range of $4000-500 \mathrm{~cm}^{-1}$ at a resolution of $\pm 1 \mathrm{~cm}^{-1}$. Variable temperature measurements were made using a specially constructed devise which was calibrated for temperature control prior to each measurement.

\section{Results and Discussion}

3.1. Room Temperature FT-Infrared Spectroscopy. The infrared spectra for all compounds are typical for metal carboxylates; that is, they are composed of low, high, and medium intensity bands, distributed between 3500 and $500 \mathrm{~cm}^{-1}$. In fact, their spectra can be divided into three main regions: the methyl and methylene group stretching region $\left(2840-2980 \mathrm{~cm}^{-1}\right)$, the carboxylate region $\left(1565-1470 \mathrm{~cm}^{-1}\right)$, and the low frequency region between 1470 and $500 \mathrm{~cm}^{-1}$, generally termed as the "methylene progression region." However, this region represents more than just the $\mathrm{CH}_{2}$ progression and is very important (vide infra).

Vibrations associated with the asymmetric $\left(\nu_{\mathrm{as}}\right)$ and symmetric $\left(\nu_{\mathrm{s}}\right)$ carboxyl (COO) stretching modes (Figure 1(a)) are located in the regions of $\sim 1565$ and $\sim 1420 \mathrm{~cm}^{-1}$, respectively. These bands, formed as a result of complete resonance in the carboxyl moieties due to coordination with the metal, show significant structural sensitivity; hence, they are extensively used in the literature for the characterization of metal-carboxyl coordination and symmetry [11-15]. For example, the difference in stretching frequencies between these two vibrations, $\Delta v=v_{\mathrm{as}}-v_{\mathrm{s}}$, is related to the type of 


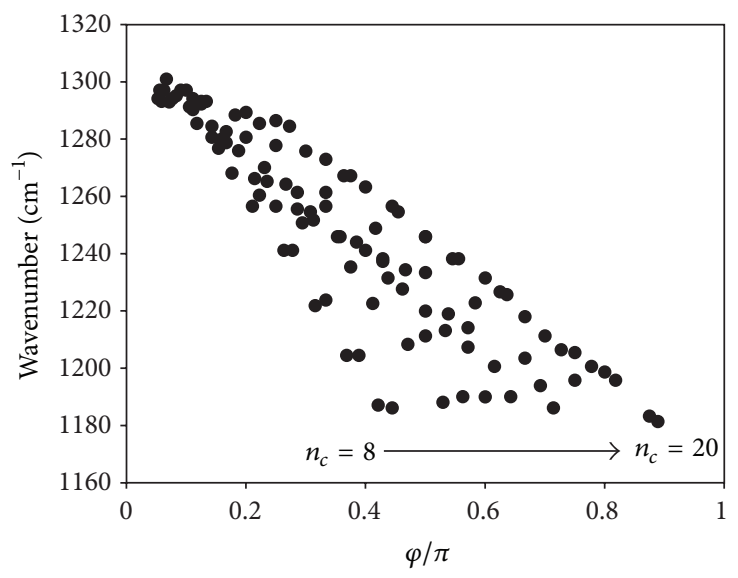

(a)

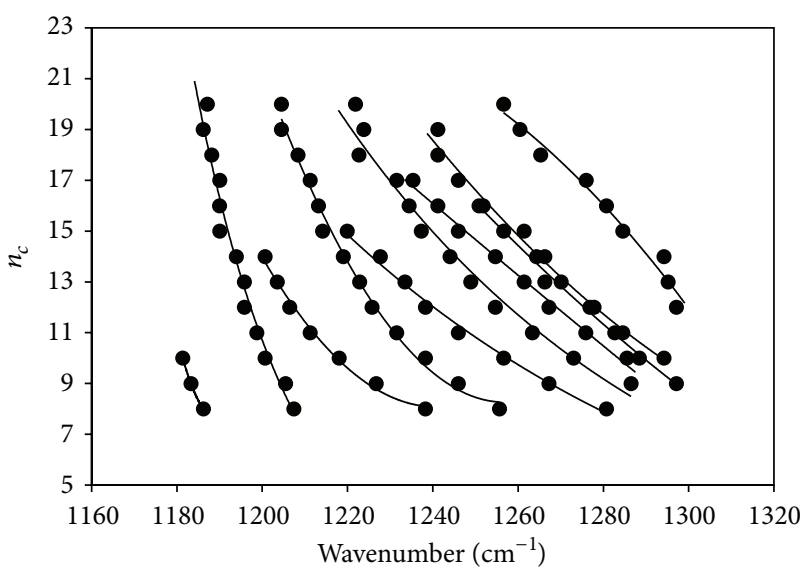

(b)

Figure 2: (a) Frequency phase curves for the methylene twisting-rocking $\left(\tau-\delta \mathrm{CH}_{2}\right)$ modes for all chain lengths $\left(\mathrm{AgC}_{8} \cdots \mathrm{C}_{20}\right.$, inclusive $)$ at room temperature. (b) Room temperature array of the methylene twisting-rocking bands $\left(\tau-\delta \mathrm{CH}_{2}\right)$ bands for silver(I) $n$-alkanoates $\left(\mathrm{AgC}_{8} \cdots \mathrm{C}_{20}\right.$, inclusive $)$.

metal-carboxyl bonding; that is, if $\Delta \nu \approx 100 \mathrm{~cm}^{-1}$, chelating coordination is indicated but for those in the region of 150 to $170 \mathrm{~cm}^{-1}$, bridging bidentate bonding is likely [17-19]. However, values in excess of $200 \mathrm{~cm}^{-1}$ indicate that the $\mathrm{C}$-O bonds are highly asymmetric; thus coordination is monodentate. The presence of extensive intermolecular interactions in the vicinity of the carboxyl group reduces the accuracy of this type analysis [20]. Hence, though $\Delta v$ for silver carboxylates is in the range of $97-101 \mathrm{~cm}^{-1}$, crystal structure data, for the short adducts, show that metal-carboxyl bonding is bridging bidentate with extensive intercarboxyl interactions [5]. Interestingly, X-ray powder data showed that their coordination is independent of chain length [12]; that is, they are isostructural where metal-carboxyl bonding is concerned. The greater intensity of the $v_{\mathrm{as}} \mathrm{COO}$ relative to the $v_{\mathrm{s}} \mathrm{COO}$ band is indicative of nonequivalent $\mathrm{C}-\mathrm{O}$ bonds, possibly due to different degrees of interaction with the metal ion; that is, metal-carboxyl bonding is asymmetric. Surely, such bonding arrangement at the carboxyl groups would result in chain tilting as was concluded by Binnemans et al. [11], based on synchrotron data and Nelson $[12,14]$ and based on X-ray diffraction.

Splitting of the carboxyl bands, as observed, is indicative of reduced $\mathrm{COO}$ symmetry; hence, energetic separation of degenerate $\mathrm{COO}$ vibrational modes persists as the selection rules governing the excitation of these vibrational modes break down. Indeed, this effect is also encouraged by coupling interactions between the $\mathrm{C}-\mathrm{O}$ vibrations and the stretching mode of the adjacent carbon-carbon bond (C-COO) which bears significant double bond character as a result of resonance in the adjacent carboxyl group. In addition to the $v_{\mathrm{as}} \mathrm{COO}$ and $v_{\mathrm{s}} \mathrm{COO}$ bands, this region also consists of another structure sensitive vibration in the form of a sharp medium intensity peak, centered at $c a .1472 \mathrm{~cm}^{-1}$, associated with methylene $\left(\delta \mathrm{CH}_{2}\right)$ bending mode. For instance, splitting of this band due to intermolecular interactions between closely spaced alkyl chains is observed for monoclinic packing whilst a sharp band is indicative of triclinic arrangement where such interactions are less significant [17, 21]. Interestingly, for the compounds discussed here, this band is not split (Figure 1) but is quite broad (Full width half maximum, FWHM: $>10 \mathrm{~cm}^{-1}$ ) with a low frequency shoulder. This indicates that the silver salts reported here are most likely monoclinic as previously reported based on X-ray powder diffraction data $[12,22]$.

The neighboring region, $\sim 1400$ to $700 \mathrm{~cm}^{-1}$, consists of several bands associated with the combination vibrations of the polymethylene chain. These bands are highly sensitive to alkyl chain structure and packing; hence, a detailed description of their most informative features is presented (vide infra). Furthermore, since the phase properties of metal carboxylates and other amphiphilic systems are controlled by chain packing and structure, a basic knowledge of the features of these bands is of high importance [23-25]. Subdivision of this region reveals the methylene twisting-rocking, $\tau-\delta \mathrm{CH}_{2}$ $\left(1175-1300 \mathrm{~cm}^{-1}\right)$ and rocking-twisting $\left(700-1060 \mathrm{~cm}^{-1}\right)$ modes, henceforth referred to as the twisting and rocking modes, respectively, to avoid confusion. This region also consists of the $\mathrm{CH}_{2}$ wagging bands: $1170-1370 \mathrm{~cm}^{-1}$. However, unlike $n$-paraffins, where intensity alternation allows for differentiation between the overlapping wagging and twisting modes, the bands of this region are of very low intensities; hence, they are difficult to characterize for silver(I) $n$ carboxylates. Indeed, similar observations were reported by Snyder and Schachtschneider [26], for long chain $n$-paraffins; however, some amount of characterization was still possible. The array of methylene twisting vibrations (Figure 2(b)), for silver carboxylates, shows decreased frequencies with increased chain length, a change which is more drastic for shorter chain adducts and for the high frequency bands of this region. This is possibly due to the gradual decrease in alkyl chains polarization. Hence, for shorter chain compounds, where the chains are more polar due to closer proximity of the $\mathrm{CH}_{2}$ groups to the $\mathrm{COO}$, that is, larger $\mathrm{COO}: \mathrm{CH}_{2}$ ratios, alkyl chain vibrations are more dependent on the structure 
of the carboxyl group. Such vibrational interference between the $\mathrm{COO}$ and $\mathrm{CH}_{2}$ modes can alter $-\mathrm{CH}_{2}-\mathrm{CH}_{2}$ - coupling and, hence, lattice packing. However, assuming that this effect is minute and that the $\mathrm{CH}_{2}$ groups behave like linearly coupled identical oscillators $(m)$ with a single degree of freedom, the simple coupled oscillator theory (SCO) can be applied as was possible for $n$-paraffins [26]. This model shows that the phase difference between adjacent oscillators $\left(\varphi_{k}\right)$ is given by $\varphi_{k}=k \pi /(m+1), k=1,2,3, \ldots, m$. Hence, the vibration frequencies $\left(v_{k}\right)$ of $\delta-\tau \mathrm{CH}_{2}$ motions are controlled primarily by $\varphi_{k}$; therefore, assigning values of $k$ such that all bands fit to a similar curve yields Figure 2(a) which gives information about $\mathrm{CH}_{2}-\mathrm{CH}_{2}$ coupling changes with chain length. Interestingly, the bands for all compounds fit to separate curves unlike for $n$-paraffins where a single curve was observed. This suggest that the addition of a single methylene group to the alkyl chain results in significant changes in their coupled vibrations and hence molecular packing. This effect is reflected in their total molar enthalpies where the $\mathrm{CH}_{2}$ contribution is exponentially related to the number of methylene groups [14]. The presence of the COO group significantly affects the polarity of the alkyl chains and hence the observed changes in their vibrational frequencies and coupling interactions with increased chain length. Such significant chain length dependence of this effect is strongly reflected in $\varphi_{k}$; hence, it is different for all compounds (Figure 2(a)). Clearly, the phase differences are more significant for longer chain adducts most likely because the $\mathrm{CH}_{2}$ groups are less affected by the carboxyl group and are therefore more independent in their vibrational motions. This assertion is supported by the convergence of $\varphi_{k}$ above $1280 \mathrm{~cm}^{-1}$ where the COO group influence is quite significant. Furthermore, solid state ${ }^{13} \mathrm{C}-\mathrm{NMR}$ data [12] shows clearly that the electron density distribution (polarity) of silver carboxylates is highly chain length-dependent; that is, the influence of the COO group decreases with increased chain length.

The carbon-carbon stretching $(\nu \mathrm{C}-\mathrm{C})$ bands, for silver carboxylates, at $c a .1115 \mathrm{~cm}^{-1}$, are among the most intense in this region, occurring at a frequency similar to that for lead(II) [27], $1128 \mathrm{~cm}^{-1}$, and cerium(III) [28], 1106-1112 $\mathrm{cm}^{-1}$, $n$-alkanoates. Interestingly, for silver $n$-eicosanoate, this band exists as a sharp singlet, at $c a .1114 .8 \mathrm{~cm}^{-1}$, but becomes increasingly broad with decreasing chain length; that is, a low frequency shoulder centered at $c a .1108 \mathrm{~cm}^{-1}$ begins to emerge for $n_{c}<18$. At $n_{c}=16$, this band is completely split with components at $\sim 1115$ and $\sim 1099 \mathrm{~cm}^{-1}$. This is a clear indication of changes in the alkyl chain electron density distribution and hence structure and packing with chain length. However, such subtle changes were not observed in reported $\mathrm{X}$-ray data [12]. However, solid state ${ }^{13} \mathrm{C}-\mathrm{NMR}$ results showed clear changes in their electron densities with increased chain length; that is, the long chain homologues were composed of more carbons that were electronically indistinguishable compared to the shorter chain adducts.

The region of $700-1060 \mathrm{~cm}^{-1}$ consists of several low intensity bands, primarily associated with the rocking mode of the methylene groups $\left(\rho \mathrm{CH}_{2}\right)$. However, it should be noted that none of these bands can be completely designated as purely rocking since there is always contamination from the twisting modes, an effect which decreases monotonically with decreasing frequency. Hence, the band of highest intensity, centered at $c a .718 \mathrm{~cm}^{-1}$, present for polymethylene chains with more than four successive $\mathrm{CH}_{2}$ groups, represents the purest form of $\rho \mathrm{CH}_{2}$ motion and is commonly referred to as the $\mathrm{CH}_{2}$ rocking band [29]. This vibration is highly sensitive to chain packing and is therefore often used in the accurate prediction of crystal packing. For example, splitting or excessive broadening caused by close packing of adjacent alkyl chains in the lattice, as observed for silver $n$-carboxylates, suggests monoclinic or orthorhombic packing whilst a sharp band correlates with a triclinic or hexagonal crystal system [30]. Interestingly, the four additional bands associated with the $\mathrm{CH}_{2}$ rocking-twisting motion, in this region, show no obvious trend with chain length. However, according to Snyder [21], $(n-1) / 2$ and $(n-2) / 2$ bands are allowed for odd and even chain $n$-paraffins having $n$ carbon atoms, respectively, whilst for silver $n$-alkanoates a similar relationship is observed but only for the $\tau-\delta \mathrm{CH}_{2}$ bands in the region of 1175$1300 \mathrm{~cm}^{-1}$. For instance, $(n / 2)+1$ and $(n+1) / 2$ bands are allowed for odd and even chain silver $n$-carboxylates, respectively. This indicates that though the general vibrational behaviours of metal $n$-carboxylates and $n$-paraffins are similar, they are not identical; that is, the presence of the COO group and the metal significantly alters their vibrational features. Also, it should be noted that, like $n$-paraffins where the $\mathrm{CH}_{2} / \mathrm{CH}_{3}$ ratio affects the frequencies of these bands, for silver salts this effect is more significant since, in addition to this ratio, the $\mathrm{CH}_{2} / \mathrm{COO}$ ratio has a similar effect. This shows clearly that the spectral features for hydrocarbon type molecules can be significantly altered by the presence of different substituents. In fact, it was shown that structural manipulations as simple as the geographic location of a triple bond, in unsaturated zinc(II) $n$-alkanoates, resulted in significant changes in their liquid crystalline and spectral features [31].

Other sensitive bands associated with alkyl chain packing are located in the region of $2800-3000 \mathrm{~cm}^{-1}$. This region consists of four main bands ascribed to the symmetric $\left(\nu_{\mathrm{s}}\right)$ and asymmetric $\left(\nu_{\text {as }}\right)$ stretching modes of the hydrogens attached to the methyl $\left(\nu_{\text {as }} \mathrm{CH}_{3}\right)$ and methylene carbons $\left(\nu_{\mathrm{as}} \mathrm{CH}_{2}\right)$. The sensitivity of these bands to alkyl chain symmetry and intermolecular interactions are manifested as odd-even alternation in the $v_{\text {as }} \mathrm{CH}_{3}$, at $\mathrm{ca} .2955 \mathrm{~cm}^{-1}$, discussed in [12]; that is, lower frequencies were observed for odd chain compounds as a result of greater van der Waals interactions caused by more intimate interplanar packing of the alkyl chains. It was proposed, as supported by density data, that the vertical interlayer spacing was less for odd chain adducts; hence, the closely packed methyl groups are more polarized and facilitative of methyl-methylene carbonhydrogen electrostatic interactions which reduced the $\mathrm{C}-\mathrm{H}$ bond order [12-14]. This effect is also reflected in the shape of the $v_{\text {as }} \mathrm{CH}_{3}$ band where the FWHM and peak areas for odd chain adducts are only $\sim 50 \%$ of those for the even chain homologues. This is due to increased overlapping of the vibrational frequencies associated with the $\mathrm{CH}_{3}$ hydrogen(s) 


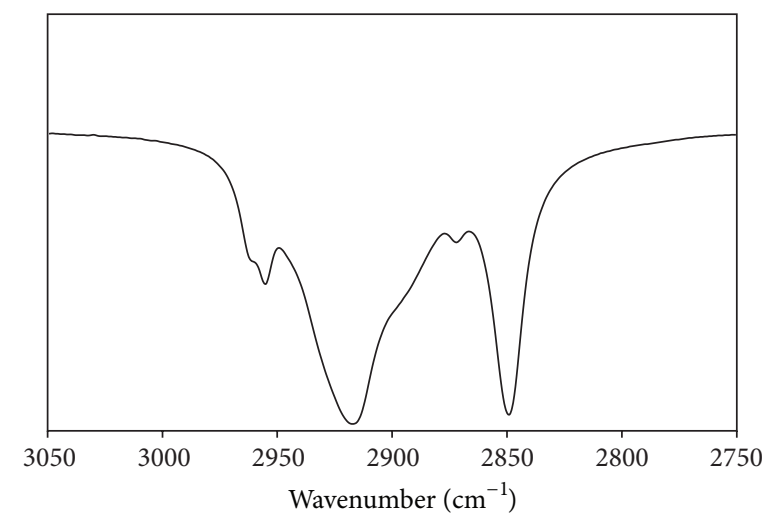

(a)

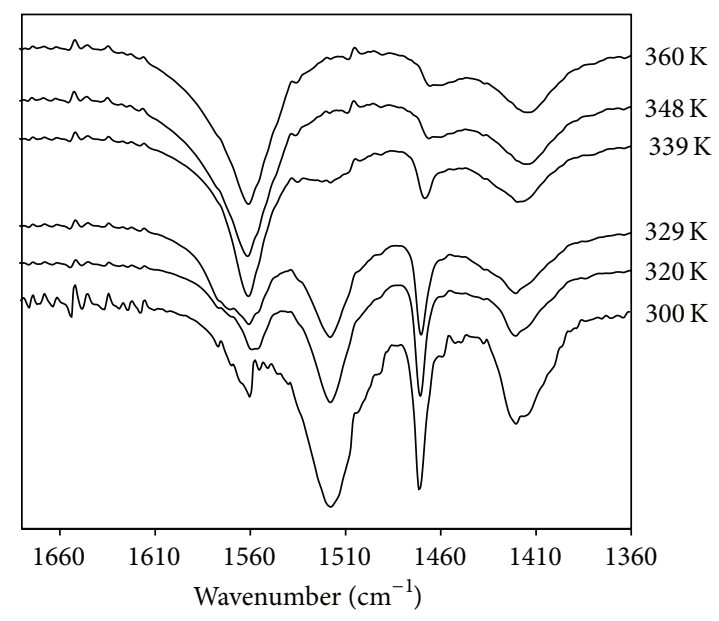

(b)

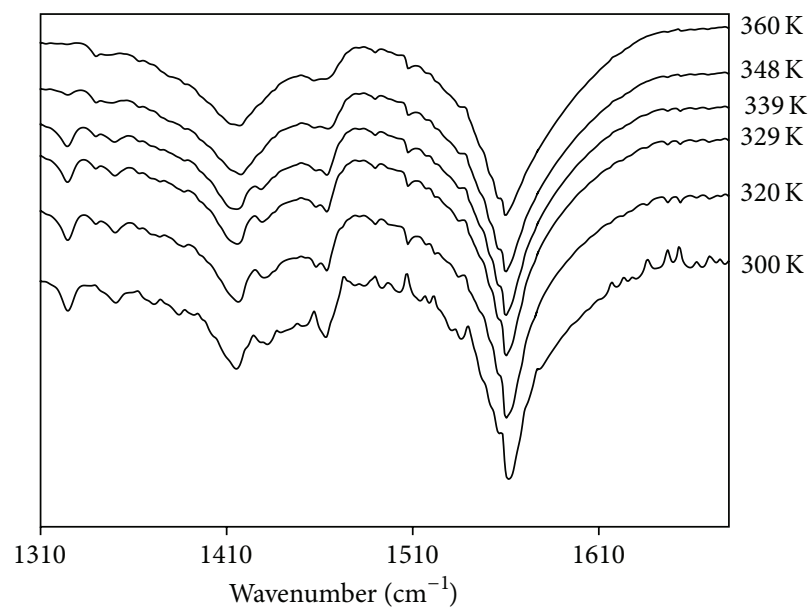

(c)

Figure 3: (a) Typical methyl and methylene group stretching region for $\mathrm{AgC}_{20}$. (b) Typical carboxyl stretching region for long $\left(n_{c}>12\right.$; $\left.\mathrm{AgC}_{20}\right)$ and (c) short $\left(n_{c}<12 ; \mathrm{AgC}_{9}\right)$ chain silver(I) $n$-carboxylate at different temperatures.

that are polarized parallel $\left(2962 \mathrm{~cm}^{-1}\right)$ and perpendicular $\left(2955 \mathrm{~cm}^{-1}\right)$ to the hydrocarbon plane. Similar frequency values, adduced from single crystals, were reported for both C-H polarization directions in $n$-paraffins [32]. The other bands in this region are associated with the $v_{\mathrm{as}} \mathrm{CH}_{2}$ and $v_{\mathrm{s}} \mathrm{CH}_{2}$ modes, at $c a .2849$ and $2917 \mathrm{~cm}^{-1}$, respectively [33-35]. Integration of these bands yields a constant relative area $\left(v_{\mathrm{as}} \mathrm{CH}_{2} / \nu_{\mathrm{s}} \mathrm{CH}_{2}\right)$ of $\sim 2.3$ which is in reasonably good agreement with that for crystalline $n$-hexane and $n$-heptane where a value of $3.0 \pm$ 0.4 was adduced based on a "simple Raman bond moment model" for tetrahedral $\mathrm{CH}_{2}$ geometry [36]. This indicates that the $\mathrm{CH}_{2}$ groups of silver(I) $n$-alkanoates are tetrahedral as expected. Comparison of the $\mathrm{CH}_{2}$ stretching frequencies for crystalline $n$-alkanes in the all-trans conformation $\left(v_{\text {as }} \mathrm{CH}_{2} \sim\right.$ 2847 and $v_{\mathrm{s}} \mathrm{CH}_{2} \sim 2916 \mathrm{~cm}^{-1}$ ) to those for silver $n$-alkanoates shows that their alkyl chains are $\sim 99.95 \%$ all-trans, at ambient temperature, and are therefore fully extended. Interestingly, like the methyl asymmetric stretching band, $v_{\text {as }} \mathrm{CH}_{2}$ is also quite broad, bearing a shoulder on both its high and low frequency sides (Figure 3(a)). The shoulder on the low frequency side corresponds accurately to the first overtone of the
$\mathrm{CH}_{2}$ bending, intensified by fermi resonance with the $v_{\text {as }} \mathrm{CH}_{2}$ vibration. However, the high frequency shoulder is probably due to correlation splitting common for orthorhombic or monoclinic crystal systems and should be accompanied by a second weaker band of a different polarization, similar to observations reported for $n$-paraffins $[37,38]$.

3.2. Variable Temperature FT-Infrared Spectroscopy. Superambient FT-Infrared measurements show, inter alia, increased stretching frequencies, reduced intensities, disappearance, and even the emergence of new bands in the spectra for these silver(I) $n$-alkanoates. For example, the carboxyl stretching region shows significant changes with increased temperature; that is, progressive decrease in both $v_{\text {as }} \mathrm{COO}$ and $v_{\mathrm{s}} \mathrm{COO}$ bands is observed, especially for long chain adducts (Figure 3(b)). However, beyond $338 \mathrm{~K}$ there is no further decrease in the intensity of the $v_{s} \mathrm{COO}$ band though the $v_{\text {as }} \mathrm{COO}$ peak has almost completely disappeared at this temperature (Figure 3(b)). Interestingly, simultaneous with the decrease in intensity of the low frequency component of the $v_{\mathrm{as}} \mathrm{COO}$ vibration, there is significant increase in the intensity 
of the band at $1560 \mathrm{~cm}^{-1}$, the high frequency component of this vibrational mode. Surely, this is indicative of significant bonding and symmetry changes in the metal-carboxyl coordination sphere. Furthermore, since $\Delta v$ has increased, it is reasonable to propose that metal-carboxyl bonding tends towards monodentate coordination with increased temperature. This idea is also supported by the significant reduction and near disappearance of the $v_{\mathrm{s}} \mathrm{COO}$ band, a change which correlates with reduced coordination number of the metal. However, though short chain adducts also show noteworthy changes in their COO vibrations (Figure 3(c)) and eventually decompose at the melt [11-14], the fact that both $\mathrm{COO}$ stretching modes are preserved, with intensities similar to room temperature, even at $360 \mathrm{~K}$, it is clear that their degradation mechanisms and/or energetics are different from the long chain homologues. It is possible that, for these chain lengths, metal-carboxyl interaction is more rigid as is common for the short chain compounds; hence, their bonding changes occur at higher temperatures as do their melts $[11,12,15]$.

These observations correlate with reports, by various authors [11, 15], showing that on heating, long chain silver carboxylates undergo significant irreversible changes. Such changes eventually lead to decomposition at the melt-to-yield silver nanoclusters [15]. Indeed, this indicates that they could find application as precursors in the solid state thermal synthesis of nanoparticles for various applications. Furthermore, if methods reported by Hermans and coworkers [39] for the synthesis of dimetal carboxylates are employed, the formation of silver dimetal salts of the form RCOOAg $\mathrm{M}_{x}$ could be possible, thermal decomposition of which could yield mixed metal quantum dots. Also, since long and short chain silver carboxylates behave differently at elevated temperatures, it is possible that their decomposition products are different. Unfortunately, there are no comprehensive reports where the decomposition mechanism and kinetics of a homologous series of silver carboxylates are clearly established.

In addition to changes in the carboxyl absorptions, the methylene bending mode, at $1472 \mathrm{~cm}^{-1}$, shows a significant decrease in intensity with increased temperature, for both long and short chain adducts (Figures 3(b) and 3(c)). This is indicative of significant changes in their lattice packing as the hydrocarbon chains become increasingly molten and gauchetrans isomerization becomes more pronounced. This assertion is supported by significant reduction in the intensities of $\mathrm{CH}_{2}$, wagging, twisting, and rocking bands, all of which nearly disappearances completely above $350 \mathrm{~K}$. The C-C stretching bands at $~ 1114$ and $1096 \mathrm{~cm}^{-1}$ also show significant reduction in their intensities as well as complete disappearance of at least one component without coalescence. In fact, at high temperatures, the $1114 \mathrm{~cm}^{-1}$ band becomes increasingly broad, indicating that even at high temperatures there is still distinct carbon environment present in the alkyl chain as discussed (vide supra). The principal $\mathrm{CH}_{2}$ rocking band centered at $\sim 719 \mathrm{~cm}^{-1}$ shows a $\sim 97 \%$ decrease in intensity (Figure 4(b)), in addition to disappearance of the shoulder observed at room temperature for long chain adducts but for short chain compounds its shape is preserved. This suggests that the melting behaviour for long and short chain compounds is not identical; hence, different numbers of phase transitions have been reported for them [13]. The decrease in intensity of the $\mathrm{CH}_{2}$ rocking band is also an indication of reduced crystalline order but interestingly, the fact that the features of this band which correlates with monoclinic packing (splitting), at ambient temperature, changed to that for triclinic packing (no splitting); it is clear that the melting mechanism, for long chain compounds, involves structural transition from a monoclinic to a thermally induced triclinic state. However, for short chain compounds this transitions is not present; that is, their room temperature structures are more stable than for the long chain homologues. Extrapolation of the intensity-temperature variation of this peak shows that it should be almost zero at $370 \mathrm{~K}$, a temperature corresponding to the range for the first transition for compounds with 8 to 15 carbon atoms, inclusive. This indicates that the first transition for these short chain compounds is associated, mainly, with alkyl chain melting and reduced crystal system order. However, for long chain adducts, at this temperature, the monoclinic arrangement and crystal system order are almost totally lost due to their greater thermal sensitivity.

The high frequency bands ascribed to the $\mathrm{C}-\mathrm{H}$ symmetric and asymmetric stretching vibrations of the $\mathrm{CH}_{2}$ and $\mathrm{CH}_{3}$ groups also show significant temperature sensitivity (Figure 4(a)). For instance, both $v_{\mathrm{s}} \mathrm{CH}_{3}$ and $v_{\text {as }} \mathrm{CH}_{3}$ bands, at ca. 2874 and $2955 \mathrm{~cm}^{-1}$, respectively, decrease simultaneously in intensity with increased temperature, indicating increased rotational freedom of the methyl and methylene groups due to reduce van der Waals interactions as the alkyl chains become increasingly molten. Coalescence of the parallel and perpendicularly polarized methyl $v_{\text {as }} \mathrm{C}-\mathrm{H}$ modes correlate with polarization isotropy and reduced interplanar van der Waals interactions due to increased $\mathrm{CH}_{3}$ rotational freedom. Similar changes in the $\mathrm{CH}_{2}$ stretching bands with increased temperature provide significant insights into the thermally induced structural and conformational evolution of the methylene group population and hence the alkyl chains. For example, increased temperature results in marked reduction of both $v_{\mathrm{as}} \mathrm{CH}_{2}$ and $v_{\mathrm{s}} \mathrm{CH}_{2}$ intensities due to reduce conformation order of the alkyl chains. Interestingly, intensity ratios of these peaks, in Raman, associated with the $\mathrm{C}-\mathrm{H}$ stretching vibrations, are known to be sensitive to the alkyl chain conformational order and environment [40-43]. Hence, a basic quantitative model allowing assessment of the lateral chain packing order(S) of alkyl chain was defined: $S=(r-0.7) / 1.5$, where $r$ is the intensity ratio of $\mathrm{CH}_{2}$ asymmetric $\left(\mathrm{I}^{-}\right)$: symmetric $\left(\mathrm{I}^{+}\right)$stretching modes, so that $S$ is unity for crystalline alkyl chains and zero for those in the liquid state [44]. This method has been applied in the structural studies of cholesteric liquid crystals [40] and biomembranes [41, 42]. However, for these compounds, a plot $S$ versus temperature gave a positive slope which would indicate that the alkyl chains become more orderly with increased temperature, an obviously incorrect result. This indicates that the model does not hold true for silver carboxylates. Nonetheless, since the ratio of $\mathrm{CH}_{2}\left(\mathrm{I}^{-} / \mathrm{I}^{+}\right)$ increases with temperature (Figure 4(c)), it is clear that the percentage gauche conformation in the methylene group 


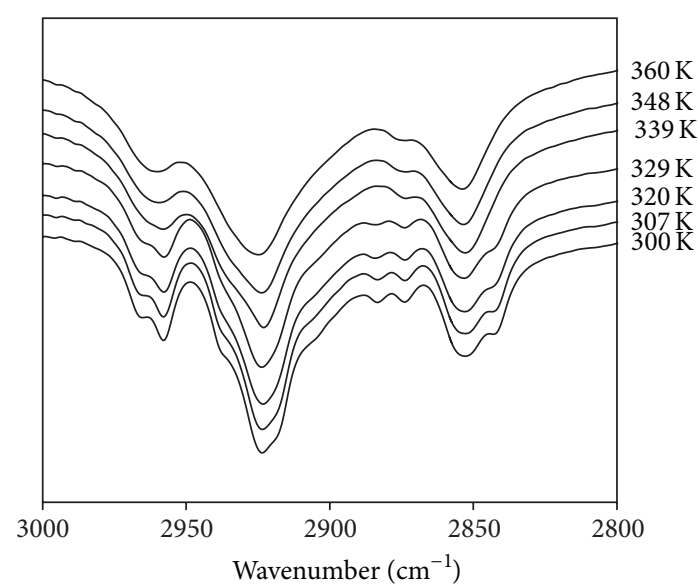

(a)

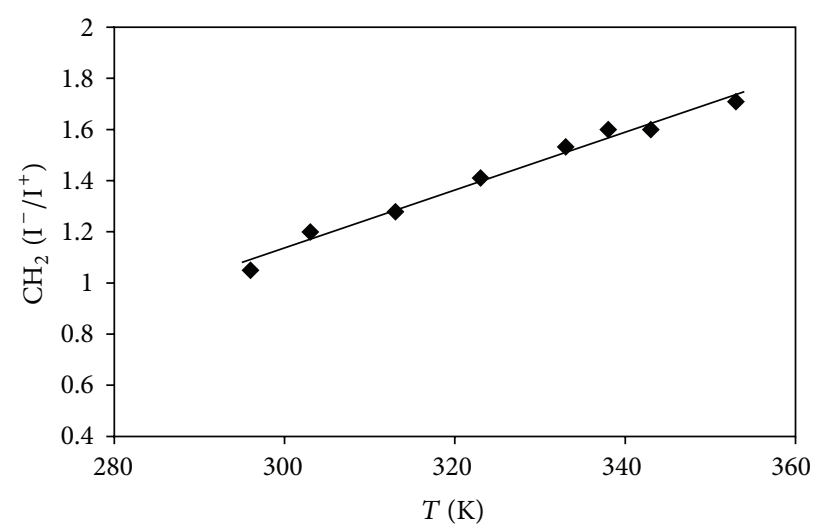

(c)

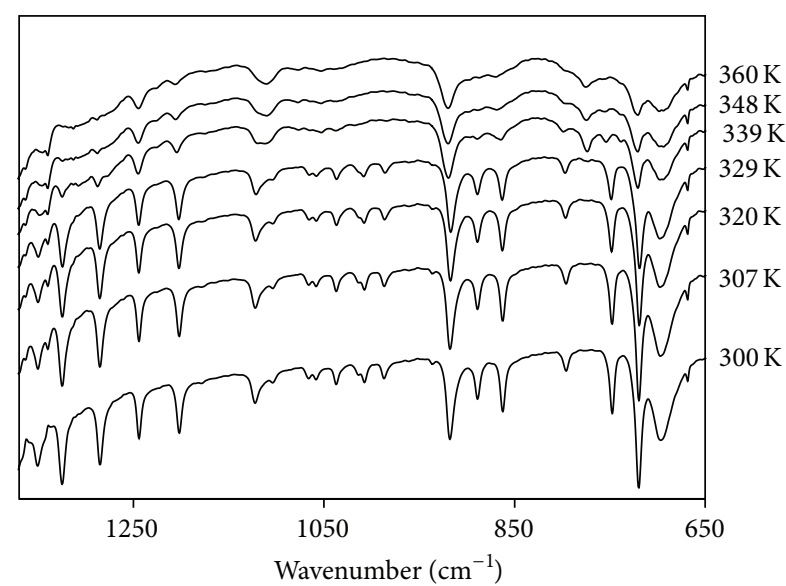

(b)

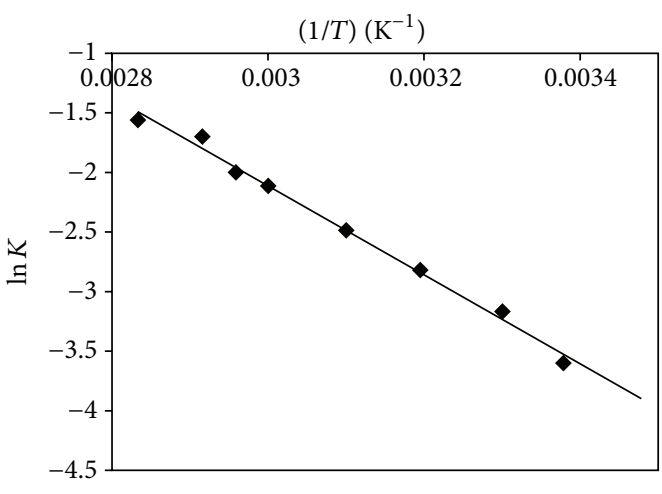

(d)

Figure 4: (a) Typical methyl and methylene stretching region and (b) methylene progression region at different temperatures. (c) Methylene asymmetric : symmetric stretching band intensity ratios versus temperature (equation: $y=0.0113 x-2.2597, R^{2}=0.9843$ ). (d) von t' Hoff plot for the effect of temperature on the trans $\rightleftarrows$ gauche interconversion equilibrium constant $K$ (equation: $y=-3727.5 x-9.0684, R^{2}=0.9927$ ).

assembly follows the same trend, as expected, and hence the observed increased asymmetric stretching probability.

Based on Figure 4(c), the intensities of both bands should be equal at $c a .289 \mathrm{~K}$; that is, lateral packing is optimum in this temperature range: the alkyl chains are $100 \%$ all-trans. The slope of this plot represents the temperature coefficient of lateral chain packing order: $\partial S / \partial T=1.13 \times 10^{-2} \mathrm{~K}^{-1}$ or the thermal sensitivity of chain packing. The magnitude of this parameter describes the strength of the intermolecular forces associated with lattice stability; that is, larger values should result for low melting point alkyl chain assemblies: weak interchain van der Waals forces; hence, this value should be much smaller for short chain compounds. In addition to reduced intensities, these bands also show changes in their frequencies; that is, with increased temperature both $v_{\text {as }} \mathrm{CH}_{2}$ and $v_{\mathrm{s}} \mathrm{CH}_{2}$ bands shift to higher frequencies. This is due to increased gauche conformation population and hence chain kinking. Indeed, the gauche population can be assessed at different temperatures by comparing the stretching frequencies of all-trans crystalline $n$-alkanes to these compounds. Therefore, if Trans $\leftrightarrows$ Gauche isomerization is viewed as a reversible equilibrium, then an estimation of the equilibrium constant $(K)$ at different temperatures based on

$$
K=\frac{1-\left(n \text {-alkane } / v_{\mathrm{s}} \mathrm{CH}_{2}\right)}{\left(n \text {-alkane } / v_{\mathrm{s}} \mathrm{CH}_{2}\right)}
$$

is possible. Therefore, a plot of $\ln K$ versus $1 / T\left(\mathrm{~K}^{-1}\right)$ (Figure $4(\mathrm{~d})$ ) gives the average isomerization enthalpy and entropy as $30.99 \mathrm{~kJ} \mathrm{~mol}^{-1}$ and $75.39 \mathrm{~J} \mathrm{~mol}^{-1} \mathrm{~K}^{-1}$, respectively. Surely, these values are not identical over different segments of the melting process as result of heat capacity changes in the methylene group assembly, especially during phase transitions where the density of the lattice changes significantly due to reduce chain packing order. This type of assessment is only valid over small temperature intervals. It is also worthy of note that the magnitude of such energy changes is affected by intermolecular forces of interactions in the lattice. By the fact that these values are large relative to those reported for sodium and potassium carboxylates [14, 22, 24], greater van der Waals interactions are indicated. However, higher melting temperatures for sodium and potassium carboxylates 
are due to greater electrostatic forces associated with the metal-carboxyl bonding.

Infrared measurements collected on cooling the samples show considerable changes at room temperature. For instance, though the methylene rocking band, centered at $c a$. $720 \mathrm{~cm}^{-1}$, at room temperature, returns to its original intensity, there is a slight shift to lower frequency $\left(\sim 717 \mathrm{~cm}^{-1}\right)$. The $\mathrm{C}-\mathrm{C}$ stretching and methylene twisting bands also show considerable changes; coalescence and broadening are most obvious, along with reduced intensities. Surely, this confirms that, on heating, even without reaching the melt, the structures of these compounds are permanently changed especially where metal-carboxyl coordination is concerned. For example, both COO modes, on cooling, do not reappear; that is, the changes observed on heating are permanent. Reemergence of the methyl and methylene $\mathrm{C}-\mathrm{H}$ stretching bands on cooling, though not totally, indicates that despite significant changes in the metal-carboxyl coordination sphere, the alkyl chains remain intact up to $360 \mathrm{~K}$. However, it is imaginable that as temperature tends towards the melt, the aforementioned changes will become more significant as proven by García and coworkers [23], for potassium $n$-carboxylates.

\section{Conclusion}

Fourier transform infrared studies on a homologous series of anhydrous solid state silver(I) $n$-carboxylates show clearly that at room temperature their methylene groups are crystallized in the all-trans conformation; hence, the alkyl chain is fully extended. The monoclinic crystal system is composed of tilted alkyl chains anchored to silver ions via bridging bidentate coordination. All bands associated with the methylene and carboxyl group vibrations show significant sensitivity to temperature, whilst the effects of chain length are mainly observable in the methylene group vibrations. Increased temperature results in reduced lateral chain packing order due to increased concentration of alkyl chain gauche conformers; that is, chain kinking arises and crystal system order diminishes. In addition to these events, there are significant irreversible bonding changes in the metal-carboxyl coordination sphere; that is, rearrangements in the bonding structure and or bonding breaking occur with increased temperature. However, the effects of temperature on lattice packing, crystalline order and metal-carboxyl bonding are somewhat different for the short chain adducts ( $<12$ carbons); that is, as chain length decreases, their thermal stability increases. Based on these results, it is clear that the molecular packing for anhydrous silver(I) $n$-alkanoates changes with increased chain length; hence, compounds of different chain length are similar but not identical.

\section{Conflict of Interests}

The author declares that there is no conflict of interests regarding the publication of this paper.

\section{Acknowledgment}

Thanks are due to Professor Henry Ellis, formerly of the University of the West Indies-Mona, for moral support whilst carrying out this work.

\section{References}

[1] F. Lacouture, M. François, C. Didierjean, E. Roccaa, J.-P. Rivera, and J. Steinmetza, "Anhydrous lead(II) heptanoate," Acta Crystallographica C, vol. 57, pp. 530-531, 2001.

[2] F. J. M. Casado, M. R. Riesco, M. V. G. Perez, M. I. Redondo, S. Lopez-Andres, and J. A. R. Cheda, "Structural and thermodynamic study on short metal alkanoates: lithium propanoate and pentanoate," Journal of Physical Chemistry B, vol. 113, no. 39, pp. 12896-12902, 2009.

[3] L. Jongen, K. Binnemans, and C. Görller-Walrand, Structure and thermal behaviour of lanthanide(III) soaps [Ph.D. thesis], Katholieke Universiteit Leuven, Leuven, Belgium, 2002.

[4] L. P. Olson, D. R. Whitcomb, M. Rajeswaran, T. N. Blanton, and B. J. Stwertha, “The simple yet elusive crystal structure of silver acetate and the role of the Ag-Ag bond in the formation of silver nanoparticles during the thermally induced reduction of silver carboxylates," Chemistry of Materials, vol. 18, no. 6, pp. 16671674, 2006.

[5] I. Weissbuch, J. Majewski, K. Kjaer, J. Als-Nielsen, M. Lahav, and L. Leiserowitz, "Mixed monolayers for the design of structured surfaces to induce oriented 3-D crystallization," Journal of Physical Chemistry, vol. 97, no. 49, pp. 12848-12857, 1993.

[6] T. C. Huang, H. Toraya, T. N. Blanton, and Y. Wu, "X-ray powder diffraction analysis of silver behenate, a possible lowangle diffraction standard," Journal of Applied Crystallography, vol. 26, no. 2, pp. 180-184, 1993.

[7] C. Zou, M. R. V. Sahyun, B. Levy, and N. Serpone, "Mechanisms of latent image formation in photothermographic silver imaging media," Journal of Imaging Science and Technology, vol. 40, no. 2, pp. 94-103, 1996.

[8] M. R. V. Sahyun, “Thermally Developable Photographic Materials (TDPM): a review of the state-of-the-art in mechanistic understanding," Journal of Imaging Science and Technology, vol. 42, no. 1, pp. 23-30, 1998.

[9] I. Geuens and I. Vanwelkenhuysen, "Physical characterization of silver behenate as a tool for the development of thermographic and photothermographic materials," Journal of Imaging Science and Technology, vol. 43, no. 6, pp. 521-527, 1999.

[10] P. M. Zavlin, A. N. Batrakov, P. Z. Velinzon, S. I. Gaft, and L. L. Kuznetsov, "Thermally developed photographic materials based on silver organic salts," Journal of Imaging Science and Technology, vol. 43, no. 6, pp. 540-544, 1999.

[11] K. Binnemans, R. Van Deun, B. Thijs, I. Vanwelkenhuysen, and I. Geuens, "Structure and mesomorphism of silver alkanoates," Chemistry of Materials, vol. 16, no. 10, pp. 2021-2027, 2004.

[12] P. N. Nelson and H. A. Ellis, "Structural, odd-even chain alternation and thermal investigation of a homologous series of anhydrous silver(I) n-alkanoates," Dalton Transactions, vol. 41, no. 9, pp. 2632-2638, 2012.

[13] P. N. Nelson, H. A. Ellis, and N. A. S. White, "Solid state ${ }^{13} \mathrm{C}-\mathrm{NMR}$, infrared, X-ray powder diffraction and differential thermal studies of the homologous series of some monovalent metal ( $\mathrm{Li}, \mathrm{Na}, \mathrm{K}, \mathrm{Ag}) n$-alkanoates: a comparative study," Spectrochimica Acta Part A: Molecular and Biomolecular Spectroscopy, vol. 145, pp. 440-453, 2015. 
[14] P. N. Nelson and H. A. Ellis, Structure and Thermal Behaviours of $\mathrm{Ag}(\mathrm{I}), \mathrm{Na}(\mathrm{I})$ and $\mathrm{K}(\mathrm{I}) \mathrm{n}$-Alkanoates, edited by: L. Thompson, Lambert Academic Publishing, 2014.

[15] S. J. Lee, S. W. Han, H. J. Choi, and K. Kim, "Structure and thermal behavior of a layered silver carboxylate," Journal of Physical Chemistry B, vol. 106, no. 11, pp. 2892-2900, 2002.

[16] J. Berson, D. Burshtain, A. Zeir, A. Yoffe, R. Maoz, and J. Sagiv, "Single-layer ionic conduction on carboxyl-terminated silane monolayers patterned by constructive lithography," Nature Materials, vol. 14, no. 6, pp. 613-621, 2015.

[17] P. N. Nelson and R. A. Taylor, "Theories and experimental investigations of the structural and thermotropic mesomorphic phase behaviors of metal carboxylates," Applied Petrochemical Research, vol. 4, no. 3, pp. 253-285, 2014.

[18] H. Li, W. Bu, W. Qi, and L. Wu, "Self-assembled multibilayers of europium alkanoates: structure, photophysics, and mesomorphic behavior," Journal of Physical Chemistry B, vol. 109, no. 46, pp. 21669-21676, 2005.

[19] E. F. Marques, H. D. Burrows, and M. da Graça Miguel, "The structure and thermal behaviour of some long chain cerium(III) carboxylates," Journal of the Chemical SocietyFaraday Transactions, vol. 94, no. 12, pp. 1729-1736, 1998.

[20] D. A. Edwards and R. N. Hayward, "Transition metal acetates," Canadian Journal of Chemistry, vol. 46, no. 22, pp. 3443-3446, 1968.

[21] R. G. Snyder, "Vibrational spectra of crystalline n-paraffins: II. Intermolecular effects," Journal of Molecular Spectroscopy, vol. 7, no. 1-6, pp. 116-144, 1961.

[22] P. N. Nelson, Structure and Phase Properties of Some Monovalent Metal n-Alkanoates, edited by I. Oaserele, Lambert Academic Publishing, 2015.

[23] M. V. García, M. I. Redondo, and J. A. R. Cheda, “Temperature dependence of the vibrational spectra of potassium soaps: fourier transform infrared study," Vibrational Spectroscopy, vol. 6, no. 3, pp. 301-308, 1994.

[24] P. N. Nelson and R. A. Taylor, "Powder X-ray diffraction, infrared and ${ }^{13} \mathrm{C}$ NMR spectroscopic studies of the homologous series of some solid-state zinc(II) and sodium(I) n-alkanoates," Spectrochimica Acta A, vol. 138, pp. 800-806, 2015.

[25] P. N. Nelson and H. A. Ellis, "Odd-even chain packing, molecular and thermal models for some long chain sodium(I) n-alkanoates," Journal of Molecular Structure, vol. 1075, pp. 299310, 2014.

[26] R. G. Snyder and J. H. Schachtschneider, "Vibrational analysis of the n-paraffins-I: assignments of infrared bands in the spectra of $\mathrm{C}_{3} \mathrm{H}_{8}$ through n- $\mathrm{C}_{19} \mathrm{H}_{40}$, 'Spectrochimica Acta, vol. 19, no. 1, pp. 85-116, 1963.

[27] M. S. Akanni, E. K. Okoh, H. D. Burrows, and H. A. Ellis, "The thermal behaviour of divalent and higher valent metal soaps: a review," Thermochimica Acta, vol. 208, pp. 1-41, 1992.

[28] A. M. Giroud-Godquin, "My 20 years of research in the chemistry of metal containing liquid crystals," Coordination Chemistry Reviews, vol. 178-180, no. 2, pp. 1485-1499, 1998.

[29] H.-W. Li, H. L. Strauss, and R. G. Snyder, "Differences in the IR methylene rocking bands between the crystalline fatty acids and n-alkanes: frequencies, intensities, and correlation splitting," Journal of Physical Chemistry A, vol. 108, no. 32, pp. 6629-6642, 2004.

[30] J. R. Nielsen and R. G. Hathaway, "Infrared spectra of normal paraffins in the hexagonal crystal form," Journal of Molecular Spectroscopy, vol. 10, no. 1-6, pp. 366-377, 1963.
[31] P. N. Nelson, R. A. Taylor, and H. A. Ellis, "The effects of molecular and lattice structures on the thermotropic phase behaviour of zinc(II) undecanoate and isomeric zinc(II) undecynoates," Journal of Molecular Structure, vol. 1034, pp. 75-83, 2012.

[32] R. F. Holland and J. R. Nielsen, "Infrared spectra of single crystals: part I. Orthorhombic $n-\mathrm{C}_{24} \mathrm{H}_{50}$, monoclinic $n-\mathrm{C}_{36} \mathrm{H}_{74}$, and triclinic $n-\mathrm{C}_{18} \mathrm{H}_{38}$ and $n-\mathrm{C}_{20} \mathrm{H}_{42}$," Journal of Molecular Spectroscopy, vol. 8, no. 1-6, pp. 383-405, 1962.

[33] N. B. Colthup, L. H. Daly, and S. E. Wiberley, Introduction to Infrared and Raman Spectroscopy, Edited by N. L. Alpert, Academic Press, New York, NY, USA, 1975.

[34] W. E. Keiser and H. A. Szymanski, IR: Theory and Practice of Infrared Spectroscopy, Plenum, New York, NY, USA, 1973.

[35] L. J. Bellamy, The Infrared Spectra of Complex Molecules, John Wiley \& Sons, New York, NY, USA, 1975.

[36] R. G. Snyder, "Group moment interpretation of the infrared intensities of crystalline $n$-paraffins," The Journal of Chemical Physics, vol. 42, no. 5, p. 1744, 1965.

[37] S. Krimm, C. Y. Liang, and G. B. B. M. Sutherland, "Infrared spectra of high polymers. II. Polyethylene," The Journal of Chemical Physics, vol. 25, article 549, 1956.

[38] R. G. Snyder, S. L. Hsu, and S. Krimm, "Vibrational spectra in the $\mathrm{CH}$ stretching region and the structure of the polymethylene chain," Spectrochimica Acta Part A: Molecular Spectroscopy, vol. 34, no. 4, pp. 395-406, 1978.

[39] J. J. Hermans, K. Keune, A. van Loon, R. W. Corkery, and P. D. Iedema, "The molecular structure of three types of longchain zinc(II) alkanoates for the study of oil paint degradation," Polyhedron, vol. 81, pp. 335-340, 2014.

[40] B. J. Bulkin and K. Krishnan, "Vibrational spectra of liquid crystals. III. Raman spectra of crystal, cholesteric, and isotropic cholesterol esters, 2800-3100-cm-1region," Journal of the American Chemical Society, vol. 93, no. 23, pp. 5998-6004, 1971.

[41] B. J. Bulkin and N. Krishnamachari, "Vibrational spectra of liquid crystals. IV. Infrared and Raman spectra of phospholipidwater mixtures," Journal of the American Chemical Society, vol. 94, no. 4, pp. 1109-1112, 1972.

[42] K. G. Brown, W. L. Peticolas, and E. Brown, "Raman studies of conformational changes in model membrane systems," Biochemical and Biophysical Research Communications, vol. 54, no. 1, pp. 358-364, 1973.

[43] K. Larsson and R. P. Rand, "Detection of changes in the environment of hydrocarbon chains by raman spectroscopy and its application to lipid-protein system," Biochimica et Biophysica Acta-Lipids and Lipid Metabolism, vol. 326, no. 2, pp. 245-255, 1973.

[44] B. P. Gaber and W. L. Peticolas, "On the quantitative interpretation of biomembrane structure by Raman spectroscopy," Biochimica et Biophysica Acta (BBA)_Biomembranes, vol. 465, no. 2, pp. 260-274, 1977. 

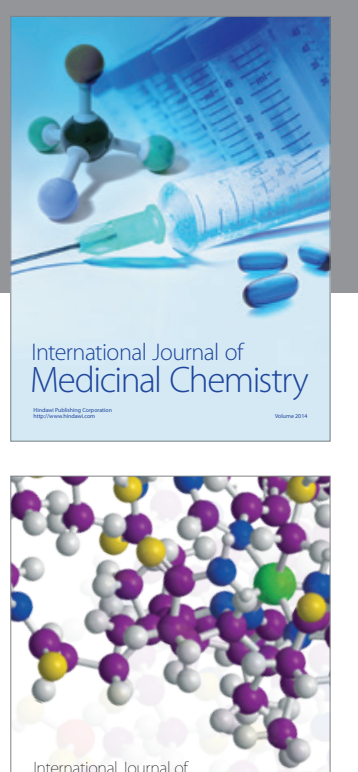

Carbohydrate Chemistry

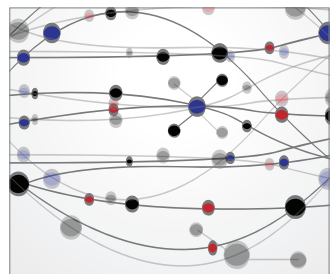

The Scientific World Journal
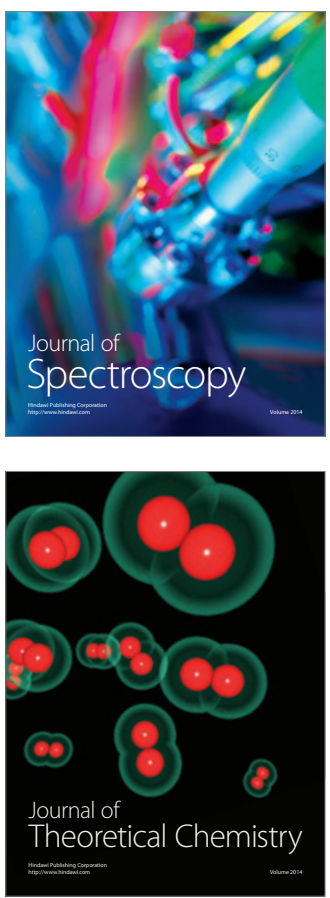
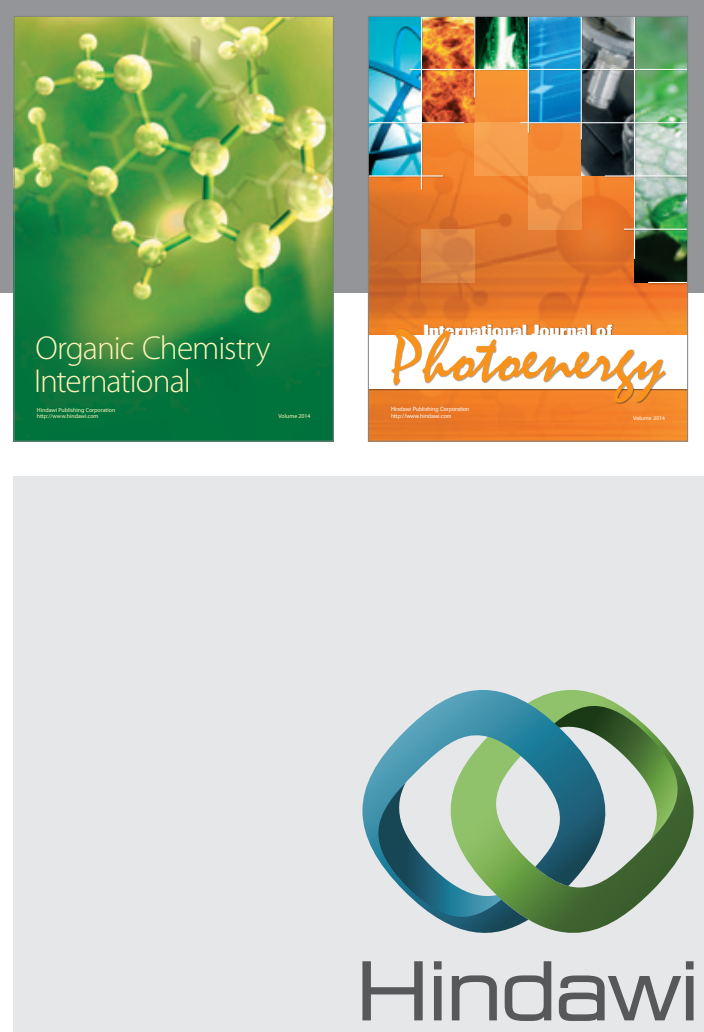

Submit your manuscripts at

http://www.hindawi.com

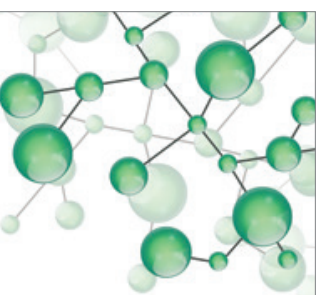

International Journal of

Inorganic Chemistry

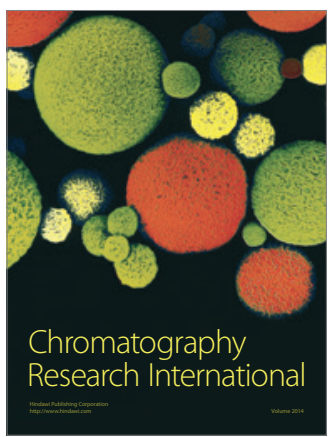

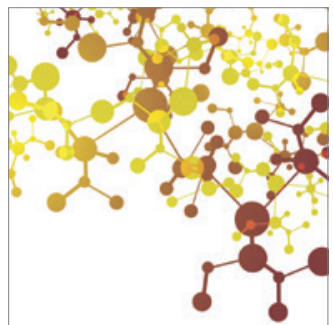

Applied Chemistry
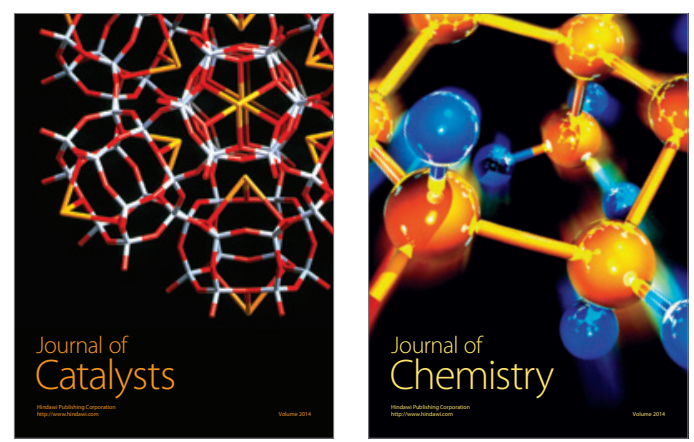
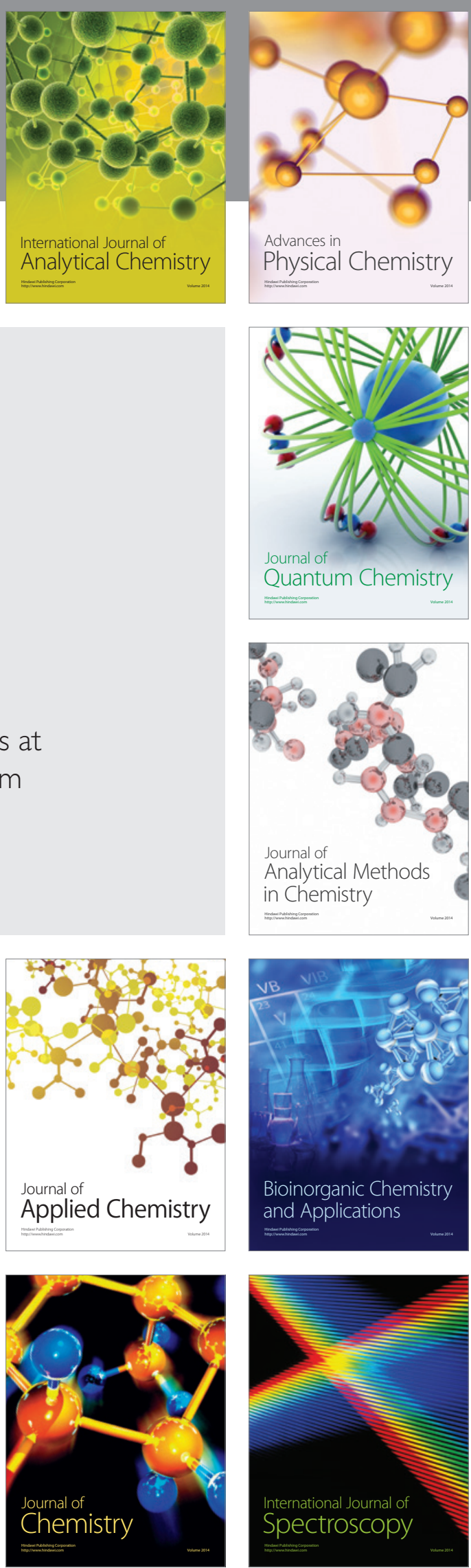